\title{
ORIGINAL
}

\section{VALIDEZ DEL PROTOCOLO DE ADECUACIÓN DE URGENCIAS HOSPITALARIAS}

\author{
Teresa Sempere Selva (1), Salvador Peiró (2), Pilar Sendra Pina (1), Consuelo Martinez Espín (3) \\ e Inmaculada López Aguilera (1). \\ (1) Servicio de Urgencias, Hospital General de Elche. \\ (2) Fundación Instituto de Investigación en Servicios de Salud. \\ (3) Servicio de Urgencias, Hospital Morales Meseguer de Murcia.
}

\section{RESUMEN}

Fundamento: Diversos trabajos sitúan la proporción de visitas inadecuadas a los servicios de urgencia hospitalarios entre el $20 \%$ y el $80 \%$ del total. Este amplio intervalo se debe, en buena parte, a la inexistencia de un consenso en torno a la definición de urgencia y el nivel asistencial idóneo para la atención de cada situación posible que conduce al uso de juicios subjetivos para evaluar la inadecuación. El objetivo de este trabajo es desarrollar y validar un instrumento objetivo de identificación de urgencias hospitalarias inadecuadas.

Método: A partir de un marco conceptual que incluía como dimensiones la gravedad clínica del paciente, la intensidad de los servicios prestados y algunas situaciones que justificarían la adecuación de las visitas espontaneas, se construyó el Protocolo de Adecuación de Urgencias Hospitalarias, un instrumento con criterios explícitos para evaluar la adecuación de las visitas a los servicios de urgencia hospitalarios y se evaluó, en una muestra aleatoria de 100 historias clínicas de urgencias, su fiabilidad intra y entre observadores y su validez de criterio y predictiva frente al juicio de expertos.

Resultados: El Protocolo de Adecuación de Urgencias Hospitalarias mostró una excelente fiabilidad tanto intra como entre observadores (índices de acuerdo observado entre el $99 \%$ y $100 \%$; estadístico kappa entre 0,97 y la unidad), y validez de criterio en el límite entre moderada y baja (índice de acuerdo observado $68 \%$, estadístico kappa 0,39. Este bajo nivel de acuerdo se debe a que el Protocolo de Adecuación de Urgencias Hospitalarias se comporta como un instrumento muy especifico (los casos inadecuados según el juicio clínico son evaluados como inadecuados) pero poco sensible (sólo el $59 \%$ de los casos que el Protocolo de Adecuación de Urgencias Hospitalarias consideró apropiados, fueron valorados de esta forma por los clínicos).

Conclusiones: El Protocolo de Adecuación de Urgencias Hospitalarias se comporta como un instrumento muy fiable y capaz de identificar la fracción mas claramente inadecuada de las visitas inadecuadas a los Servicios de Urgencias Hospitalarios. Estas características lo hacen especialmente útil para las comparaciones entre centros y el seguimiento a lo largo del tiempo o la monitorización de intervenciones para reducir el uso inapropiado.

Palabras clave: Urgencias hospitalarias; utilización inapropiada; revisión de utilización.

Correspondencia:

Salvador Peiró

Fundación Instituto de Investigación en Servicios de Salud

Plaza María Beneyto 2, 10

46008 Valencia

Correo electrónico: speiro(a)comv.es

\section{ABSTRACT}

\section{The Validity of the Hospital Emergency Suitability Protocol}

Background: Different studies rate the proportional number of visits unsuited to the HER services at $20 \%-80 \%$ of all. This wide range is due, in good part, to no consensus existing as to the definition of the term (emergency" and the ideal degree of assistance for dealing with each possible situation which leads to the use of subjective judgments for evaluating unsuitability. The purpose of this study is that of developing and validating an objective tools for pinpointing unsuitable hospital emergencies.

Method: Based on a conceptual framework which included as dimensions the seriousness of the clinical condition of the patient in question, the intensity of the services rendered and some situations which would warrant spontaneous visits being suitable, the Hospital Emergency Suitability Protocol (HESP), a tool entailing explicit criteria for assessing the suitability of the visits to the HER's, and a random sample of 100 emergency room clinical histories, the reliability thereof of observers on an individual and group basis and the validity of judgment and predictive validity thereof as regarding the opinion of experts having been analyzed.

Results: The HESP revealed itself to provide an excellent reliability rate of observers on an individual and group basis (indexes of agreement fond of $99 \%-100 \%$; kappa statistic of $0.97-1.00$ ), and judgment validity on the borderline between moderate and low (index of agreement found of $68 \%$, kappa statistic 0.39). This low level of agreement is due to the fact that the HESP functions like a highly specific (the inappropriate cases accord to the clinical judgment are evaluated as inappropriate) yet not highly sensitive tool (solely $59 \%$ of the cases which the HESP considered to be suitable were evaluated as such by the clinical judgments).

Conclusions: The HESP acts as a highly reliable tool capable of pinpointing the most clearly unsuitable fraction of the inappropriate visits to the HER's. These characteristics make it useful for drawing comparisons among hospitals and for long-range follow-up or monitoring of actions for lowering the percentages of unsuitable use.

Key words: Emergencies. Inappropriate utilization. Utilization review. 


\section{INTRODUCCIÓN}

La situación de la atención en los Servicios de Urgencia Hospitalarios (SUH) es, desde hace años, objeto de preocupación para la comunidad sanitaria y la sociedad en general, ya que en todos los países desarrollados se ha producido un fenómeno de crecimiento de su utilización, el cual supuso en España pasar de 9,2 millones de visitas en 1984 a 15,3 millones en 19941. Buena parte de este incremento se atribuye a un aumento desproporcionado de pacientes que utilizan los servicios de urgencia hospitalarios (SUH) de forma inadecuada, ya sea por problemas banales o de organización de otras áreas del sistema sanitario, por problemas sociales $o$, simplemente, porque tienen más confianza en la efectividad de estos servicios que en la atención primaria.

Estudios realizados en diversos países ${ }^{2-4}$, incluido España ${ }^{5}$, sitúan el volumen de visitas inadecuadas a los SUH entre 2 y 8 de cada 10, con el resultado final de la masificación de estos servicios a causa de casos que podrían ser asistidos en atención primaria. Esta elevada utilización de los SUH para la atención de situaciones no urgentes es preocupante por varios motivos: 1) la perdida de continuidad asistencial y seguimiento de los tratamientos para los pacientes que sustituyen la atención primaria por los SUH, 2) la detracción de recursos para la atención de pacientes en situaciones de riesgo vital, 3) la sobrecarga y desorganización que trasladan al conjunto del hospital y 4) los mayores costes respecto a la atención primaria.

La mayor parte de los estudios sobre utilización inadecuada de los SUH se han realizado en un solo hospital, empleando los juicios subjetivos de los médicos de urgencias (apoyados o no en criterios guía) para evaluar la adecuación de la urgencia. En la tabla $1^{5-22}$ se muestran los resultados de los estudios realizados en España y, en su caso, los criterios adoptados para guiar esta valoración. Los resultados de visitas no urgentes van desde cifras en torno al $25 \%$ cuando se considera la utilización de pruebas diagnósticas como criterio de adecuación, hasta el $80 \%$ cuando se consideran adecuados sólo los casos que ingresaron. Esta discordancia $-\mathrm{y}$ al margen de factores de entorno y diferencias en las poblaciones incluidas- se debe fundamentalmente a la inexistencia de un consenso en torno a la definición de urgencia y, menos aún, en su operativización para decidir el nivel asistencial idóneo para cada tipo de situación ${ }^{23,24}$. A este respecto, un trabajo que utilizó 7 sistemas diferentes - previamente publicados - de clasificación de las urgencias sobre los mismos 598 pacientes, mostró proporciones de urgencias inapropiadas desde el 10\% al 90\% según el sistema utilizado ${ }^{25}$. Un segundo trabajo, en el que un médico de urgencias y un internista revisaron independientemente las mismas 219 historias, halló un claro acuerdo sobre la clínica de los pacientes, pero enormes diferencias en la valoración de la condición de «urgente» y sobre el lugar adecuado para su tratamiento ${ }^{26}$. En este estudio no se halló correlación entre las valoraciones de cualquiera de los clínicos y las percepciones de los pacientes. Un tercer estudio halló sólo un acuerdo moderado entre las valoraciones de 2 métodos de triage y el juicio de los clíni$\cos ^{27}$.Esta situación se traduce en valoraciones fundamentalmente subjetivas, incluso cuando se cuenta con criterios guía, y en la imposibilidad de comparar la posición relativa de hospitales diferentes o de seguir la evolución de un hospital a lo largo del tiempo.

El objetivo de este trabajo es, precisamente, desarrollar y validar un instrumento objetivo de identificación de urgencias hospitalarias inadecuadas. Este objetivo suponía, básicamente, 1) definir un marco conceptual que incluyera una definición operativa de urgencia hospitalaria, sus dimensiones y la población de aplicación; 2) valorar la validez de contenido (operativización de los conceptos y dimensiones en criterios objetivos) y someter el resultado a valoración de expertos (validez aparente); 
Tabla 1

Porcentaje de urgencias hospitalarias inadecuadas y criterios de valoración de la adecuación en estudios en España

\begin{tabular}{|c|c|c|}
\hline Autor & $\begin{array}{l}\% \text { Inade- } \\
\text { cuación }\end{array}$ & Criterio de definición de la urgencia hospitalaria \\
\hline Castillo M, 1986 (6) & 58,6 & $\begin{array}{l}\text { Necesidad de control exhaustivo y constante, de tratamientos complejos, de explo- } \\
\text { raciones complementarias, y de visita por médicos especialistas }\end{array}$ \\
\hline Muiño $A, 1988(7)$ & 37 & $\begin{array}{l}\text { Sintomatología intensa de reciente comienzo, procesos diagnosticados extrahospi- } \\
\text { talariamente que requieren atención especializada urgente, necesidad de explora- } \\
\text { ciones complementarias no diferibles en su realización, descompensación en pa- } \\
\text { ciente con grave enfermedad de base }\end{array}$ \\
\hline Balanzó X, 1989 (8) & 78,9 & $\begin{array}{l}\text { No justificada: la que desde ningún punto de vista precisa asistencia urgente intra } \\
\text { o extrahospitalaria. Ligeramente grave: cuando necesita una visita medica priori- } \\
\text { taria pero sin el concurso o técnicas hospitalarios. }\end{array}$ \\
\hline Diego F, $1990(9)$ & 35,5 & No especifica criterios (juicio subjetivo) \\
\hline Ibáñez I, 1991 (10) & 44,9 & $\begin{array}{l}\text { Necesidad de ingreso, de tratamiento urgente o exploraciones complementarias no } \\
\text { disponibles con carácter inmediato en AP, de asistencia por un médico especialista no } \\
\text { disponible en el momento en AP, signos y sintomas que sugieran al paciente peligro } \\
\text { para su vida o que en otras ocasiones hubieran precisado asistencia hospitalaria. }\end{array}$ \\
\hline Rodríguez Gutierrez, 1992 (11) & 65 & $\begin{array}{l}\text { Urgencia extrahospitalaria: podría ser atendida por un médico y diagnosticada con } \\
\text { analitica básica y } \mathrm{Rx} \text { simple. Urgencia hospitalaria: precisa atención urgente no } \\
\text { incluida en el apartado anterior. No Urgente: No precisa atención sanitaria o ésta } \\
\text { no puede ser englobada en las definiciones anteriores }\end{array}$ \\
\hline Antón MD, 1992 (12) & 65,0 & $\begin{array}{l}\text { Urgencia no hospitalaria: necesidad de atención inmediata, sin que exista peligro } \\
\text { para la vida y no pueda esperar a ser visto por su pediatra o médico de cabecera en } \\
\text { cl horario habitual de consulta. No urgente: cualquicr otra situación }\end{array}$ \\
\hline Alonso M, 1993 (13) & 47,9 & $\begin{array}{l}\text { Situaciones que por su gravedad, complicaciones potencialmente graves o por la } \\
\text { necesidad de exploraciones complementarias que no estuvieran al alcance de la } \\
\text { AP requirieran los recursos del hospital. }\end{array}$ \\
\hline Cubero $P, 1994(14)$ & 60 & $\begin{array}{l}\text { Pacientes remitidos por un médico, situaciones que precisan asistencia hospitala- } \\
\text { ria urgente, situaciones que sin requerir atención hospitalaria inminente son vivi- } \\
\text { das como urgencias por el paciente o sus familiares. }\end{array}$ \\
\hline Marco MT, 1994 (15) & 55 & $\begin{array}{l}\text { Ingreso hospitalario, estancia en sala de observación, pruebas complementarias } \\
\text { inexistentes en las urgencias de AP }\end{array}$ \\
\hline Descarrega $\mathrm{R}, 1994$ (16) & - & Igual que Ibáñez, 1989. \\
\hline González-Grajera B,1995 (17) & 49,5 & $\begin{array}{l}\text { Ingreso hospitalario, permanencia en observación } 12 \text { horas o más, interconsulta } \\
\text { con especialista, alta voluntaria contra el criterio médico, traslado a otro hospital, } \\
\text { fallecimiento durante la estancia en el hospital. }\end{array}$ \\
\hline Lapeña 1996 (18) & 69 & $\begin{array}{l}\text { Urgencia hospitalaria: emergencia vital o necesidad de medios diagnósticos no dispo- } \\
\text { nibles en AP. Urgencia no hospitalaria: necesidad de atención inmediata, sin peligro } \\
\text { para la vida pero que no puede ser demorada. No Urgente: cualquier otra situación }\end{array}$ \\
\hline Sansa Pérez L, 1996 (19) & $56-72$ & Juicios subjetivos, incluyendo motivos clínicos y sociales. \\
\hline Llorente S, $1996(20)$ & 24,1 & Igual que Ibáñez, 1989. \\
\hline Escobedo F, 1997 (21) & 54,7 & Igual que Ibáñcz, 1989 , más valoración previa como urgente por un médico. \\
\hline Oterino D, $1999(22)$ & 26,8 & Protocolo con criterios explícitos (PAUH) modificado. \\
\hline Sempere $T, 1999(5)$ & 29,6 & Protocolo con criterios explícitos (PAUH). \\
\hline
\end{tabular}

AP: atención primaria; PAUH: Protocolo de adecuación de urgencias hospitalarias. 
3) valorar la aplicabilidad del instrumento (aceptabilidad, tiempo de administración y coste); 4) valorar la fiabilidad entre e intraobservadores; y 5) valorar la validez de criterio y predictiva respecto a un patrón de referencia.

\section{MATERIAL Y MÉTODO}

\section{Marco conceptual}

Inicialmente, se conceptualizaron como inapropiadas aquellas visitas a los SUH, que habiendo sido derivadas por un médico o espontaneas, por su gravedad y/o por la atención prestada se podrían haber atendido en otro nivel asistencial. Este marco puede visualizarse mediante una tabla de doble entrada en función de la urgencia y el nivel de atención requerido que daría lugar a 4 situaciones posibles: urgencias hospitalarias, urgencias de atención primaria, atención de nivel hospitalario pero no urgente, y atención no urgente resoluble en atención primaria.

El análisis preliminar de este marco dejaba entrever grandes dificultades para separar rígidamente los niveles asistenciales de cada tipo de urgencia. De un lado, el nivel resolutivo de la atención primaria podía variar en diferentes lugares, horas del día, laborales y festivos y otras situaciones; de otro lado, la evaluación se convertiría en un juicio a posteriori (una vez conocida la gravedad del paciente y los cuidados y pruebas que ha requerido) de una decisión de derivación (o del paciente) tomada sin esta información. Igualmente, en determinadas situaciones al menos, atender las urgencias primarias en los SUH podía ser una estrategia razonable (por ejemplo, en los horarios nocturnos de poca afluencia, puede ser más eficiente atender las urgencias primarias en los SUH -dado que de todas formas deben permanecer abiertos, el coste de la atención a estos casos se limitaría a los costes variables - que abrir nuevos servicios que incluyan costes estructurales). Además, se consi- deró que identificar todo este tipo de situaciones obligaría al diseño de un instrumento muy complejo, repleto de condicionales, que impedirían algunas de las características buscadas: sencillez, rapidez en la valoración, poca ambigüedad y aplicación a partir de información usual y disponible en la historia clínica de urgencias.

Todas estas circunstancias llevaron a una modificación de los planteamientos iniciales en el sentido de que el instrumento debería maximizar la clasificación como adecuadas de las urgencias de nivel hospitalario (especificidad), aún a costa de perder sensibilidad y clasificar como adecuadas parte de las urgencias resolubles en atención primaria y situaciones que requieren atención hospitalaria, pero no urgentes. La población diana se estableció en los pacientes mayores de 14 años que acuden a los SUH de hospitales de agudos, excluyendo la obstetricia, y se consideró el uso del instrumento para mediciones de grupo (no para mediciones individuales), tanto de forma concurrente como retrospectiva a través de la revisión de la historia clínica de urgencias, pero no el uso prospectivo como instrumento de triage.

A partir este marco, se valoraron como dimensiones de interés: 1) la gravedad clínica del paciente, 2) la intensidad de los servicios prestados tratamientos, pruebas diagnósticas, necesidad de observación prolongada o ingreso- y su habitual disponibilidad inmediata en atención primaria, y 3) algunas situaciones particulares que justificarían la adecuación de las visitas espontaneas.

\section{Diseño del cuestionario y validez de apariencia}

A partir de la definición de estas dimensiones se realizó una extensa revisión de la literatura y, en especial, de los instrumentos y criterios diseñados para la identificación retrospectiva de la adecuación de urgen$\operatorname{cias}^{28-37}$, de ingresos hospitalarios inapropia- 
$\operatorname{dos}^{38-40}$ así como instrumentos de triage utilizados por diversos autores ${ }^{25-27,41-52}$. A partir de la selección de criterios definidos en estos trabajos y de la revisión de la literatura española ${ }^{6-21}$ se confeccionaron las versiones preliminares del Protocolo de Adecuación de Urgencias Hospitalarias (PAUH). Sobre estas versiones se realizó un trabajo de eliminación de criterios redundantes, revisiones y recogida de comentarios y sugerencias por clínicos experimentados en urgencias hospitalarias y atención primaria, y se revisaron diversos manuales de urgencias para verificar que ningún proceso potencialmente grave quedaba sin identificar como adecuado, ya fuera a partir de los síntomas (gravedad) o de los tratamientos y pruebas diagnósticas usuales en estos casos. En concreto se verificó la identificación como adecuadas de las urgencias debidas a problemas cardio-circulatorios (paro cardíaco, infarto de miocardio, síndromes isquémicos sin infarto, arritmias cardiacas, edema pulmonar, crisis hipertensiva, embolia pulmonar, embolismo periférico, disección aórtica, taponamiento cardíaco), respiratorias (insuficiencia respiratoria, crisis asmática, neumotórax, atelectasia pulmonar, obstrucción laríngea aguda, derrame pleural), digestivas (hemorragia digestiva alta, abdomen agudo, íleo, insuficiencia hepática aguda), renal (insuficiencia renal aguda), endocrinológicas y del metabolismo (descompensación diabética, hipoglucemia, crisis addisoniana, crisis tireotóxica, hipercalcemia, tetania), vásculo-cerebrales y ncurológicas (accidente vásculo-cerebral, isquemia transitoria, lipotimias, convulsiones, compresión espinal, polineuritis ascendente) y otras (miastenia gravis y crisis colinérgica, tétanos, intoxicaciones, psicosis aguda, delirium tremens, shock anafiláctico, shock séptico, fracturas). La versión final del PAUH (tabla 2) está constituida por 5 apartados que incluyen criterios de gravedad, tratamiento, pruebas diagnósticas, resultados y un último apartado aplicable sólo a pacientes que acudieron al SUH sin ser derivados por un médico. Los criterios 1.1 a 1.8 responden a la valoración de gravedad y se centran, sobre todo, en la estabilidad de los sistemas fisiológicos (pulso, presión, temperatura, equilibrio electrolítico, gases) y en la pérdida brusca de funcionalidad de algún órgano o sistema (incluyendo fracturas, hemiplejías, etcétera).

El segundo bloque de criterios (2.1 al 2.4) se basa en la realización en urgencias de tratamientos que se relacionan con la gravedad o cuya realización en atención primaria - aún no imposible - puede ser dificultosa en algunos momentos. Además de los procedimientos realizados en quirófano y la puesta de yesos, se incluye la administración de oxigeno, de cualquier fármaco por vía intravenosa y de fluidos cuando se indican con cualquier finalidad que no sea el mantener una vía de forma preventiva. El tercer bloque de criterios (3.1 a 3.4) se fundamenta en la realización de pruebas diagnósticas en urgencias que orientan hacia la necesidad de hacer un diagnóstico rápido. Al emplear estos criterios (al igual que los criterios de tratamiento) se asume la necesidad de realizar tales procesos de forma urgente $\mathrm{y}$, por tanto, se evalúa la adecuación frente a las actuaciones reales en urgencias y no frente a un estándar ideal de lo que debería haberse hecho. Esto es, si en el SUH se ha solicitado una prueba radiológica o de laboratorio urgente porque se consideró que el paciente la requería para su evaluación diagnóstica, se presume que el médico de atención primaria derivó de forma adecuada a urgencias. Se intentó eliminar las pruebas disponibles usualmente en atención primaria y aquellas que pueden considerarse rutinarias en algunos tipos de pacientes. También se consideraron una serie de criterios (4.1 a 4.2) como el ingreso del paciente o una estancia prolongada en urgencias, que sugieren adecuación de la derivación.

Para el supuesto de pacientes espontáneos, se desarrollaron una serie de criterios (5.1 a 5.8) que justificarían la visita a urgencias sin necesidad de una previa en atención primaria. Además de cualquiera de los criterios previos, se consideraron determinadas 
Tabla 2

Protocolo dc adecuacion de urgencias hospitalarias (PAUH)

\begin{tabular}{|c|c|}
\hline 1 & CRITERIOS DE GRAVEDAD. \\
\hline 1.1 & Perdida de conciencia, desorientación, coma, insensibilidad (brusca o muy reciente) \\
\hline 1.2 & Perdida brusca de visión o audición \\
\hline 1.3 & Alteraciones pulso $(<50 />140$ pulsaciones/minuto) y arritmias. \\
\hline 1.4 & Alteración presión arterial (sistólica: $<90 />200 \mathrm{mmHg}$; diastólica: $<60 />120 \mathrm{mmHg}$ ). \\
\hline 1.5 & $\begin{array}{l}\text { Alteraciones de electrolitos o gases sanguíneos (No considerar en pacientes con alteraciones crónicas de estos paráme- } \\
\text { tros: insuficiencia renal crónica, insuficiencia respiratoria crónica, etc.) }\end{array}$ \\
\hline 1.6 & Fiebre persistente ( 5 días) no controlada con tratamiento en atención primaria. \\
\hline 1.7 & Hemorragia activa (hematemesis, epixtasis, melenas, etc.). Excluye heridas superficiales que sólo requieren sutura. \\
\hline 1.8 & Perdida brusca de la capacidad funcional de cualquier parte del cuerpo \\
\hline 2 & CRITERIOS DE TRATAMIENTO. \\
\hline 2.1 & Administración de medicación o fluidos por vía intravenosa (excepto mantenimiento de vía) \\
\hline 2.2 & Administración de oxigeno \\
\hline 2.3 & Colocación de yesos (excluye vendajes) \\
\hline 2.4 & Intervención/procedimiento realizado en quirófano. \\
\hline 3 & CRITERIOS DE INTENSIDAD DIAGNÓSTICA: \\
\hline 3.1 & Monitorización de signos vitales o toma de constantes cada 2 horas. \\
\hline 3.2 & Radiología de cualquier tipo. \\
\hline 3.3 & $\begin{array}{l}\text { Pruebas de laboratorio (excepto glucemia en diabéticos que acuden por motivos no relacionados con la diabetes y prue- } \\
\text { bas de glucemia en tira seca). }\end{array}$ \\
\hline 3.4 & Electrocardiograma (excepto cardiopatía crónica que acude por problemas no relacionados cardiopatía). \\
\hline 4 & OTROS CRITERIOS \\
\hline 4.1 & El paciente está más de 12 horas en observación en el SUH \\
\hline 4.2 & El paciente es ingresado en el hospital o trasladado a otro hospital \\
\hline 4.3 & El paciente fallece en el SUH \\
\hline 4.9 & Otros en paciente derivado por un médico (especificar). \\
\hline 5 & CRITERIOS APLICABLES SÓLO A PACIENTES QUE ACUDEN ESPONTÁNEAMENTE \\
\hline 5.1 & Proviene de un accidente (trafico, laboral, en lugar público,...) y hay que evaluar al paciente. \\
\hline 5.2 & Sintamos que sugieren urgencia vital: dolor torácico, disnea instauración rápida, tiraje, dolor abdominal agudo. \\
\hline 5.3 & Cuadro conocido por el paciente y que habitualmente requiere ingreso. \\
\hline 5.4 & Al paciente le ha dicho un médico que acuda a Urgencias si se presenta el sintoma \\
\hline 5.5 & Requiere atención medica primaria con rapidez y el hospital es el centro más cercano \\
\hline 5.9 & Otros en pacientes espontáneos (especificar). \\
\hline
\end{tabular}

SUH: Servicio de urgencias hospitalario.

situaciones (síntomas que sugieren riesgo vital, experiencia de pacientes crónicos, indicaciones previas de médicos, accidentes, etc.) que, como norma general, justificarían que un paciente acudiera a urgencias hospitalarias. Tanto para los pacientes derivados por un médico (apartado 4.9) como para los espontáneos (apartado 5.9) se admite la posibilidad de empleo de criterios subjetivos que deben especificarse para su valoración posterior.
El cumplimiento de un solo criterio de cualquier apartado identificaría la visita en urgencias como adccuada, mientras que aquellos casos que no cumplieran ningún criterio se considerarían inadecuados. Con este diseño final, se preveía que el PAUH sería capaz de identificar como adecuadas la gran mayoría de las urgencias que realmente fueran adecuadas, aunque también identificaría como tales parte de las que son inadecuadas, especialmente cuando el criterio de adecuación deriva 
exclusivamente del cumplimiento de criterios de tratamiento y, sobre todo, diagnósticos.

El PAUH se complemento con una lista de posibles causas de visita inapropiada (tabla 3 ), construida también a partir de la revisión de la literatura y sugerencias de clínicos, que pretendía ser lo más extensa y detallada posible, y cuyo objeto es asignar causas de inadecuación a partir de la valoración subjetiva del médico de urgencias en revisiones concurrentes. Como en el caso del PAUH, se utilizan criterios para pacientes derivados por un médico y espontáneos, y se admite la posibilidad de emplear otros si son especificados.

\section{Entorno}

Servicio de urgencias del Hospital General Universitario de Elche, que cubre la asistencia en el área sanitaria 19 (Baix Vinalopó), a la que están adscritas las poblaciones de Elche (191.600 habitantes), Crevillent (23,781 habitantes) y Santa Pola (17.099 habitantes). Las urgencias están organizadas en dos áreas bien diferenciadas, la de Obstetricia-Ginecología y la de Urgencias generales. Esta última, a su vez, está dividida en: una zona para Pediatría separada del resto, y otra zona donde se atienden las urgencias de Traumatología, Cirugía, Medicina Interna y Especialidades. Durante 1996 se atendieron algo mas de 90.000 visitas de las que un tercio corresponderían a pediatría y obstetricia y de las que aproximadamente un $20 \%$ finalizaron en ingreso hospitalario.

\section{Muestra}

Se utilizó una muestra de 100 historias clínicas (HC) de urgencias, obtenidas al azar, de pacientes que acudieron al Servicio de Urgencias Generales del Hospital del Elche entre mayo del 96 y abril del 97, excluyéndose las $\mathrm{HC}$ de los pacientes que fueron atendidos en el Servicios de Pediatría (menores de 14 años), las urgencias obstétricas y las atendidas directamente por el servicio de traumatología.

\section{Proceso de revisión}

Para el análisis de fiabilidad intra-revisor, las $100 \mathrm{HC}$ fueron revisadas por uno de los autores, facultativo de urgencias del hospital, que volvió a revisarlas a los 60 días del primer examen. Para el análisis de la fiabilidad entre revisores se compararon los resultados de la primera con la realizada sobre las mismas historias por un segundo facultativo de urgencias con 9 años de experiencia en esta unidad, y previamente entrenado en el manejo del PAUH. Para evaluar la validez del PAUII, las mismas $100 \mathrm{HC}$ fueron revisadas conjuntamente por 2 facultativos del SUH, con amplia experiencia en atención de urgencias hospitalarias ( 10 y 14 años de ejercicio hospitalario), que evaluaron la necesidad de la hospitalización para cada una de estas atenciones según su propio juicio clínico. Estos revisores no recibieron ningún tipo de entrenamiento o formación previa en revisión de historias y no conocían el diseño del instrumento.

En todos los casos estuvieron disponibles y fueron utilizados todos los documentos contenidos en la historia clínica de urgencias, incluyendo los datos administrativos, el informe de alta de urgencias, el de ingreso hospitalario o, en su caso, ingreso en la unidad observación, ordenes y notas médicas, notas de enfermería y gráficas, informes de interconsulta, de pruebas radiológicas $u$ otras pruebas diagnósticas, terapéuticas y los resultados de las pruebas de laboratorio. El tiempo medio de revisión fue de 10 minutos por $\mathrm{HC}$.

\section{Análisis}

La fiabilidad del PAUH, definida como el grado de reproducibilidad de los resultados cuando el instrumento es utilizado por el mismo observador en 2 ocasiones separadas por el tiempo, o por observadores diferentes, se evaluó mediante varios índices de acuerdo entre parejas de revisores. El índice de acuerdo observado ${ }^{53,54}$ se define como la proporción de casos en los que coincide el resultado en ambas revisiones. El índice de acuerdo específico se define como la razón entre los ca- 
Tabla 3

Causas de utilización inapropiada de urgencias hospitalarias

\section{PACIENTES DERIVADOS POR UN MÉDICO}

1 No es una urgencia. No requiere atención inmediata.

1.2 El paciente requiere atención inmediata, pero resoluble fuera del hospital.

1.3 Remitido desde Consulta Externa para agilizar el diagnóstico

1.4 Remitido por error

1.9 Otros: especificar

\section{PACIENTES ESPONTÁNEOS}

2 Demora excesiva en otro dispositivo asistencial:

2.1 - Lista de espera quirúrgica

2.2 - Lista de espera de consulta externa hospitalaria

2.3 - Consulta externa hospitalaria (demora entre visitas)

$2.4 \quad$ Consulta de especialista de zona

2.5 - Consulta en atención primaria (cita previa)

2.6 - Pruebas diagnósticas solicitadas por atención primaria o especialista de zona

2.7 - Pruebas diagnósticas solicitadas por el hospital

$2.9 \quad-$ Otros: especificar

3 Fallo en la atención continuada

3.1 - La consulta del médico general ha finalizado

3.2 - Demora en visita a domicilio de atención primaria

3.3 - Demora en visita a domicilio del Servicio de Urgencias

3.4 - Imposibilidad de contactar con el centro de salud

3.5 - Imposibilidad de contactar con el Servicio de Urgencias

3.9 - Otros: especificar

$4 \quad$ El paciente no conoce como utilizar el dispositivo asistencial

4.1 - No tiene médico de cabecera asignado

4.2 - No conoce la localización/teléfono de su médico de cabecera

4.3 - No conoce la existencia/localización/teléfono del Servicio de Urgencias

4.9-Otros: especificar

5 Mayor confianza en el hospital o desconfianza en el dispositivo de atención primaria

5.1 - Ha acudido al médico de cabecera y «no se fia»

5.2 - Ha acudido al Servicio de Urgencias y «no se fia»

5.3 - Acudió directamente a Urgencias Hospitalarias.

5.4 - Tiene historia abierta en el hospital y cree que será mejor atendido.

5.9 - Otros: especificar

6 Comodidad y problcmas del paciente o su entorno:

6.1 - Vive cerca/No pierde horas trabajo/atención rápida/mas cómodo/...

6.2 - Problemas del paciente: $\mathrm{Cl}$ bajo, hipocondríaco, simulador,...

6.3 - Búsqueda de una exploración (radiología, analítica,...)

$6.4 \quad$ La familia quiere ingresar al paciente.

$6.5 \quad--$ Requerimiento de una autoridad pública: policía, juez,...

6.9 - Otros: especificar

9 Otros: especificar

sos juzgados inapropiados en 2 revisiones y el total de casos que al menos uno de los re- visores consideró inapropiado ${ }^{53-55}$. Este índice ofrece una mejor valoración que el de 
acuerdo observado en la evaluación de las urgencias inapropiadas, ya que no depende del habitualmente alto grado de acuerdo en las urgencias que son consideradas apropiadas. El estadístico kappa de Cohen ( $\kappa$ ) se utilizó para corregir el acuerdo debido al azar, permitiendo estimar la significación estadística (y los correspondientes intervalos de confianza) entre la diferencia en el grado de acuerdo que sería esperable simplemente por azar (valor 0) y el grado de acuerdo observado (el acuerdo perfecto no debido al azar recibe el valor 1). Para la interpretación de $\kappa$ se ha sugerido que valores por encima de 0,75 corresponderían a niveles de acuerdo excelentes, entre 0,75 y 0,40 corresponderían a niveles de acuerdo buenos $\mathrm{y}$, por debajo de 0,40 , representarían bajos niveles de acuerdo entre observadores ${ }^{53,56}$.

El término validez, o más estrictamente validez de criterio, se refiere a la modida en que los resultados obtenidos concuerdan con el resultado verdadero o con un «patrón oro» bien correlacionado con la variable a medir. En este estudio se evalúa mediante el grado de acuerdo entre los resultados obtenidos en la primera revisión con el PAUH y el juicio emitido por los clínicos que revisaron las historias basándose en sus propios criterios. Los índices utilizados para evaluar la validez de criterio son los mismos empleados para medir la fiabilidad (índice de acuerdo observado, de acuerdo específico y estadístico $\kappa$ ). Para evaluar la validez predictiva del PAUH se calculó su sensibilidad, especificidad y valores predictivos frente al juicio de los revisores clínicos.

\section{RESULTADOS}

En la tabla 4 se muestran los resultados del análisis de fiabilidad intra-revisor, que muestran una concordancia completa entre las 2 revisiones realizadas en diferentes momentos del tiempo. En la tabla 5 se muestran los resultados del análisis de fiabilidad entre-revisores, que presenta 1 sólo

Tabla 4

Fiabilidad intra-revisores del protocolo de adecuacion de urgencias hospitalarias

\begin{tabular}{|llccc|}
\hline & & \multicolumn{2}{c|}{ REVISOR A } & TOTAL \\
\cline { 3 - 5 } & & Apropiado & Inapropiado & 75 \\
\hline \multirow{2}{*}{ REVISOR A } & Apropiado & 75 & 0 & 25 \\
\cline { 2 - 5 } & Inapropiado & 0 & 25 & 100 \\
\hline
\end{tabular}

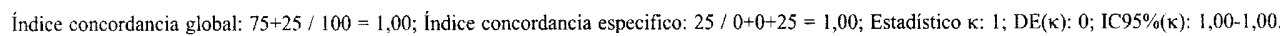

Tabla 5

Fiabilidad entre revisores del protocolo de adecuacion de urgencias hospitalarias

\begin{tabular}{|llccc|}
\hline & & \multicolumn{2}{c|}{ REVISOR B } & TOTAL \\
\cline { 2 - 4 } REVISOR A & Apropiado & 75 & 0 & 75 \\
\hline & Inapropiado & 1 & 24 & 25 \\
\hline & TOTAL & 76 & 24 & 100 \\
\hline
\end{tabular}

Índice concordancia global: $75+24 / 100=0,99 ;$ Índice concordancia especifico: $24 / 1+0+24=0,96 ;$ Estadistico $\kappa: 0,97 ; D E(\kappa): 0,03 ; \mathrm{IC} 95 \%(\kappa): 0,93-1,00$ 
caso de discordancia, con elevados índices de acuerdo: 99\% para el acuerdo observado, $96 \%$ para el acuerdo específico y kappa de 0,97 .

En la tabla 6 se muestran los resultados del análisis de validez de criterio frente al juicio de expertos. Tanto el índice de concordancia global $(68 \%)$ como el específico
(43\%) muestran niveles bajos de acuerdo y el estadístico kappa se situó en el limite entre los valores de acuerdo moderado y bajo $(0,39)$, debiéndose las discrepancias a que los revisores clínicos consideraron un 55\% de casos inapropiados frente a sólo el 24\% del PAUH. Sólo en 1 caso el PAUH consideró inapropiada una urgencia que los revisores clínicos consideraron apropiada.

Tabla 6

Validez de criterio del protocolo de adecuacion de urgencias hospitalarias frente al juicio de expertos

\begin{tabular}{|llccc|}
\hline & & \multicolumn{2}{c|}{ EXPERTOS } & TOTAL \\
\cline { 2 - 5 } & & Apropiado & Inapropiado & 75 \\
\hline \multirow{2}{*}{ REVISOR A } & Apropiado & 44 & 31 & 25 \\
\cline { 2 - 5 } & Inapropiado & 1 & 24 & 100 \\
\hline
\end{tabular}

Indice concordancia global: $44+24 / 100=0,68$; Índice concordancia especifico: $24 / 1+31+24=0,43 ;$ Estadistico $\kappa: 0,39 ; \mathrm{DE}(\kappa): 0,07 ; \mathrm{IC} 95 \%(\kappa): 0,24-0,53$.

En la tabla 7 se muestra el valor predictivo del PAUH frente al juicio de expertos. Los resultados muestran que se comporta como un instrumento específico pero poco sensible, con un elevado valor predictivo para los casos etiquetados como inapropiados (el 96\% de los casos evaluados como inapropiados por el PAUH fueron considerados inapropiados por los clínicos), pero escaso valor predictivo negativo (sólo el 59\% de los casos que el PAUH consideró apropiados, fueron valorados de esta forma por los clínicos).

Tabla 7

Validez predictiva del protocolo de adecuacion de urgencias hospitalarias frente al juicio de expertos

\begin{tabular}{|llccc|}
\hline & & \multicolumn{2}{c|}{ EXPERTOS } & TOTAL \\
\cline { 3 - 5 } REVISOR A & Inapropiado & 24 & 1 & 25 \\
\cline { 2 - 5 } & Apropiado & 31 & 44 & 75 \\
\hline & TOTAL & 55 & 45 & 100 \\
\hline
\end{tabular}

Sensibilidad: $24 / 55=0,44$; Especificidad: $44 / 45=0,98$; Valor predictivo positivo (inapropiado): $24 / 25=0,96$; Valor predictivo negativo (apropiado): $44 / 75=0,59$. 


\section{DISCUSIÓN}

El PAUH, tal y como se había hipotetizado, se comportó como un instrumento altamente fiable, con valores del estadístico $\kappa$ siempre en los rangos del acuerdo excelente ${ }^{53,54,56}$. Igualmente, los índices de concordancia global y específico fueron muy elevados, tanto en la prueba test-retest ( $100 \%$ para ambos índices), como en el análisis entre revisores (99\% y $96 \%$, para los respectivos índices de concordancia). Estos resultados son superiores a los hallados en la literatura para los instrumentos objetivos de evaluación de la inadecuación de ingresos y estancias hospitalarias como el Appropriateness Evaluation Protocol ${ }^{38,55,57-60}$, el Intensity-Severity-Discharge Criteria Set $^{55,61,62}$ o el Standardized Medreview Instrument ${ }^{55}$, probablemente porque los criterios del PAUH son menos ambiguos que algunos de los existentes en estos instrumentos.

Esta elevada fiabilidad del PAUH contrasta con la escasa reproducibilidad de los juicios subjetivos de los clínicos ${ }^{63-65}$, incluso cuando se basan en criterios guía, y era una de las características buscadas por el diseño del PAUH, con el objetivo de permitir su uso en las comparaciones entre hospitales o entre distintos momentos temporales del mismo hospital, sin que estas se vieran afectadas por la variabilidad en el uso del instrumento de evaluación.

Respecto a la validez de criterio frente al juicio de expertos, los resultados del análisis sitúan al PAUH como un instrumento de moderada a baja validez. Sin embargo, esta primera apreciación debe ser matizada ya que, excepto uno, todos los casos identificados como inapropiados por cl PAUH fueron evaluados de esta forma por los revisores clínicos, de modo que el PAUH se comporta, como evidencia el análisis de validez predictiva, como un instrumento con alta especificidad pero baja sensibilidad (siempre frente al juicio subjetivo de expertos). Hay que señalar que tras la revisión del único caso valorado como inapropiado por el PAUH frente a la valoración como apropiado de los clínicos, el mismo en el que discre- paban los revisores del análisis de fiabilidad entre-observadores, se consideró que el primer revisor había aplicado incorrectamente los criterios.

Estos resultados eran esperables dados los criterios de construcción del PAUH que buscaban maximizar la fiabilidad (la capacidad para reproducir los resultados cuando es empleado por personas diferentes o en momentos diferentes) y la especificidad (evitar etiquetar como inadecuadas las urgencias apropiadas), aspectos que se consiguieron parcialmente a expensas de sacrificar la sensibilidad e incrementar el porcentaje de falsos apropiados. Se prefirió este diseño por 4 motivos fundamentales: 1) permitir, como se ha comentado, las comparaciones entre centros; 2) evitar la perdida de valide $z$ aparente que supondría identificar urgencias adecuadas como inapropiadas, aspecto crítico para el uso y difusión del instrumento; 3 ) adoptar como referencia el estándar de práctica real y no un estándar ideal; y 4) construir un instrumento de fácil uso, con criterios sencillos y poco ambiguos, que pudieran valorarse a partir de la información usualmente disponible en urgencias. De esta forma, y como se había previsto, el PAUH clasifica como inapropiadas solamente la fracción más claramente inadecuada de las visitas a los $\mathrm{SUH}$, dejando las zonas grises bajo la etiqueta de visitas adecuadas.

En los 2 trabajos de campo que han utilizado el PAUH hasta la fecha ${ }^{5,22}$, el criterio más frecuente de considerar las visitas como adecuadas fue la realización de pruebas diagnósticas (casi dos tercios de las visitas cumplían criterios de pruebas, y alrededor del 20\% fueron consideradas adecuadas sólo por estos criterios, mientras que el resto cumplían otros criterios adicionales) siendo mucho menos frecuente el uso de criterios de gravedad (alrededor del 25\%) o intensidad del tratamiento (alrededor del 35\%). Este aspecto tiene interés para diferenciar la utilización inadecuada de los SUH por los usuarios o los médicos de atención primaria, de la utilización inadecuada de pruebas diagnósticas por los propios SUH. Así, 
mientras que los clínicos que evaluaron la validez del PAUH estimaron que solo un $45 \%$ de las visitas eran adecuadas, en la realidad solicitaron pruebas diagnósticas urgentes al $65 \%$ de los pacientes, indicando que en muchos casos los SUH actúan con los pacientes no-urgentes como si fueran urgentes. La llamativa doble valoración empleada por los clínicos que les lleva a considerar que no debería haber acudido a urgencias (o ser remitido por su médico de atención primaria) un paciente en el que ellos mismos han creído necesario realizar pruebas diagnósticas en un plazo inmediato, parece ser la principal fuente de discrepancias entre el PAUH y los juicios clínicos.

Probablemente, esta característica es la principal limitación que debe tenerse en cuenta para interpretar los resultados del PAUH, ya que lo hace sensible a la intensidad diagnóstica y terapéutica de cada SUH $y$, al extremo, un SUH que, aún inadecuadamente tratara a todos sus pacientes como urgentes, no encontraría visitas inadecuadas. Pero precisamente, esta situación pone de relieve que la adecuación de las visitas no deja de ser un juicio sobre en que nivel y con qué intensidad se quicre tratar a los pacientes y, en este terreno, el análisis de la cumplimentación de criterios del PAUH puede ser útil para evidenciar el manejo como urgencias reales de los casos no urgentes, situación que tiene implicaciones de interés para las políticas sanitarias sobre la utilización de las urgencias hospitalarias. De un lado, resultaría absurdo argüir la presencia de visitas inadecuadas (esto es, la derivación de la responsabilidad por el mal uso de los servicios hacia la atención primaria o el paciente) y, de otro, la orientación de mejora se situaría en cómo reducir la intensidad diagnóstica y terapéutica en las visitas no urgentes. Hasta cierto punto, el análisis de la cumplimentación de criterios puede sugerir volver la mirada hacia la utilización inadecuada de pruebas diagnósticas por los SUH, antes que clamar contra la poca educación sanitaria de los pacientes o el escaso papel de filtro de la atención primaria
Una segunda limitación del PAUH es que la valoración con el instrumento se realiza tras la atención del paciente, una vez que se dispone de la evaluación clínica y, en su caso, de pruebas complementarias $\mathrm{y}$, en consecuencia, no puede ser utilizado como instrumento de triage; obviamente, esto no es un obstáculo para su uso previsto (revisión de urgencias inapropiadas, identificación de causas y diseño de estrategias de corrección) pero debe ser tenido en cuenta para evitar usos abusivos del instrumento.

Entre las limitaciones del análisis de validación, habría que tener en cuenta que se ha realizado a partir de la revisión retrospectiva de las historias clínicas de urgencias y, por tanto, con la posibilidad de no haber obtenido suficiente información para cumplimentar algunos criterios, aspecto que podría suponer cambios respecto a las revisiones concurrentes donde puede obtenerse información directa del paciente. Un segundo aspecto a tener en cuenta es que, aunque el diseño del PAUII preveía la inclusión de los pacientes de traumatología, en este caso se optó por no incluirlos, aspecto que no permite valorar el comportamiento del PAUH en ellos.

Igualmente, entre las limitaciones dcl análisis hay que señalar que el «patrón oro» utilizado para validar el PAUH, el juicio clínico no cstructurado, es un estándar cuestionable, ya que los estudios de fiabilidad al respecto ${ }^{63.64}$ permiten su relativización. Probablemente, haber provisto a los clínicos con una definición de urgencia más cercana a los criterios del PAUH («lo que se trata como urgente en el SUH» antes que «lo que es urgente») hubiera permitido mayores niveles de concordancia entre el instrumento y los criterios clínicos. En todo caso, las revisiones en las que un médico emplea sus propios criterios para evaluar la adecuación de algún aspecto de la atención no se explícita qué información ha utilizado para realizar dicha evaluación, con el resultado de que la validez del método depende de los conocimientos, habilidad, criterios y posibles sesgos del revi$\operatorname{sor}^{63}$. La inexistencia de otro estándar más objetivo obligaba a utilizar la revisión no es- 
tructurada como patrón, intentando minimizar los posibles sesgos mediante el recurso a clínicos con amplia experiencia, sin interés manifiesto en el resultado y desconocedores durante la revisión de las características y usos posibles del PAUH.

Finalmente, hay que señalar que la diferente dotación de la atención primaria en algunos lugares (incluso con disponibilidad de laboratorio y radiología de urgencias) o los estilos de práctica de algunos SUH, podrían sugerir la conveniencia de adaptar localmente los criterios. Se trata de aspectos discutibles, ya que en el primer caso se presupone que el paciente debería conocer la disponibilidad tecnológica de cada centro en función de su patología y en el segundo se genera ambigüedad, al tener que decidir mediante juicios subjetivos qué pruebas y tratamientos son adecuados y cuáles no. Posiblemente, en estos casos deba recurrirse a una doble valoración con la aplicación estricta del PAUH y juicios extraordinarios que, por otra parte, ofrece valores añadidos en el análisis e interpretación de la información obtenida.

Las visitas innecesarias a los servicios de urgencia hospitalarios constituyen un importante problema para el Sistema Nacional de Salud, con implicaciones en la calidad de la asistencia y en los costes, que no sólo se mantiene sino que continúa incrementándose a pesar de las estrategias desarrolladas para su contención. Esta problemática, compartida con los sistemas sanitarios de la mayor parte de los países desarrollados, requiere el desarrollo de intervenciones y políticas prácticas para su resolución o, por lo menos, su contención y evaluar la efectividad de tales estrategias. En conjunto, los resultados de este estudio muestran al PAUH como un instrumento altamente fiable y capaz de identificar, al menos, la fracción más indiscutiblemente inadecuada de las urgencias inadecuadas. Se trata por tanto de un instrumento útil para comparar la proporción de visitas inadecuadas entre hospitales o el seguimiento de un hospital a lo largo del tiempo y evaluar el impacto de las intervenciones destinadas a reducir las visitas inadecuadas. El análisis de los criterios de adecuación puede ofrecer, además, información útil para valorar el uso innecesario de pruebas diagnósticas en los servicios hospitalarios de urgencia.

\section{BIBLIOGRAFÍA}

1. Instituto Nacional de Estadística. Encuesta de establecimientos sanitarios con régimen de internado 1994. Madrid: INE; 1998.

2. Liggins K. Inappropriate attendance at accident and emergency departments: A literature revicw. J Adv Nurs 1993;18:1141-1145.

3. Health Services Utilization and Research Commision. Reducing non-urgent use of the emergency department: a review of strategies and guide for future research. Saskatoon: Health Services Utilization and Research Commision; 1997.

4. Murphy AW. «Inappropriate» attenders at accident and emergency departments I: definition, incidence and reasons for attendance. Fam Pract 1998; 15: 23-32.

5. Sempere Selva T. Utilización inadecuada de las urgencias hospitalarias (tesis). Alicante: Universidad Miguel Hernández; 1999.

6. Castillo M, Huguet J, Bravo J, Cortada L. Estudio del área de urgencias de un hospital general. Grado de adecuación de las visitas. Med Clin (Barc) $1986 ; 87: 539-542$.

7. Muiño A, González VJ, Rodriguez E, Lázaro C, Fernández E. Asistencia en un servicio de urgencia: justificación de las visitas y adecuación de los ingresos. Rev Clin Esp 1988;182:374-378.

8. Balanzo X, Pujol F. Estudio multicéntrico de las urgencias de los hospitales generales básicos de Barcelona. Med Clin (Barc) 1989; 92:86-90.

9. Diego F, Franch J, Alvarez JC, Alvarez F, Pablo ML, Villamar J. Urgencias hospitalarias en el área sanitaria de León. Estudio de la repercusión en atención primaria. Aten Primaria 1990; 7:49-55.

10. Ibañez F, Gutiérrez B, Olaskoaga A. Estudio de la utilización de los servicios de urgencias hospitalarios por la población de un EAP: grado de adecuación. Aten Primaria 1991; 8:764-769.

11. Rodriguez Gutierrez C, Romera MT, Menéndez JJ, Losa J, Mendieta JM, Montabes E et al. Estudio de tiempos en el área de urgencias hospitalaria. Gac Sanit 1992; 6:113-116.

12. Antón DM, Peña JC, Santos R, Sempere E, Martínez J, Perula LA. Demanda inadecuada a un servicio de urgencias pediátrico hospitalario: factores implicados. Med Clin (Barc) 1992; 99:743-746. 
13. Alonso M, Hernández R, Busto F, Cueto A. Utilización de un servicio de urgencias hospitalario. Rev San Hig Pub 1993; 67:39-45.

14. Cubero P, Gálvez E, Salinero M, Abando I, Ayerbe MC. Uso injustificado del servicio de urgencias de un hospital general. Medifam 1994; 4:16-22.

15. Marco MT, Rodríguez M, Gobierno J, González T, Cabrera A, Pérez L. Accesibilidad a las urgencias pediátricas hospitalarias: distancia y medios diagnósticos. Aten Primaria 1994; 7:873-876.

16. Descarrega R, Gutiérrez C, Cruz L, López I. Análisis de la utilización inadecuada del servicio de urgencias de un hospital del tercer nivel. Aten Primaria 1994; 13(9):30-35

17. González-Grajera B, Mendoza R, Hinojosa J, Buitrago F. Adecuación de las derivaciones medicas a un servicio de urgencias hospitalario. Aten Primaria 1995; 7:433-436.

18. Lapeña S, Reguero S, García M, Gutiérrez M, Abdallah I, González H. Estudio epidemiológico de las urgencias en un hospital general. Factores implicados en una demanda inadecuada. An Esp Pediatr 1996; 44:121-125.

19. Llorente S, Alonso M, Buznego B. Papel de la atención primaria en la frecuentación del servicio de urgencias de un hospital comarcal. Aten Primaria.1996: 18:243-247.

20. Sansa Pérez L, Orus Escola T, Juncosa Font S, Barredo Hernández M, Traveria Casanova J. Frecuentación a los servicios de urgencias hospitalarios: motivaciones y características de las urgencias pediátricas. An Esp Pediatr 1996; 44: 97-104.

21. Escobedo F, González L, Salarich M, Manzano A, López I, Martín JA, Albadalejo C. Evaluación de las urgencias hospitalarias desde un área básica de salud. Aten Primaria 1997; 4:169-175.

22. Oterino de la Fuente D, Peiró S, Calvo Rico R, Sutil Murillo P, Fernández O, Pérez Bautista $G$ et al. Utilización inadecuada de un Servicio de Urgencias Hospitalario. Una evaluación con criterios explícitos. Gac Sanit 1999; en prensa.

23. Melguizo M, Prados A, Quesada F, Castillo R, Bailón E. Ordenación de las urgencias en atención primaria. Aten Primaria 1992; 9: 57-67.

24. Lowe RA, Bindman AB. Judging who needs emergency department care: a prerequisite for policy-making. Am J Emerg Med 1997; 15:133-136.

25. Lowe RA, Bindman AB. Refusing care to emergency department of patients: evaluation of published triage guidclincs. Ann Emcrg Mcd 1994; 23:377-379.

26. Foldes SS, Fischer LR, Kaminsky K. What is an emergency? The judgments of two physicians. Ann Emerg Med 1994; 23:833-840.
27. O'Brien GM, Shapiro MJ, Woolard RW, O'Sullivan PS, Stein MD. «Inappropriate» emergency department use: a comparison of three methodologies for identification. Acad Emerg Med $1996 ; 3: 252-257$.

28. Buesching DP, Jablonowski A, Vesta E, Dilts W, Runge $\mathrm{C}$, Lund $\mathrm{J}$ et al. Inappropriate emergency department visits. Ann Emerg Med 1985; 14:672-676.

29. DeAngelis C, Fosarelli P, Duggan AK. Use of the emergency department by children enrolled in a primary care clinic. Pediatr Emerg Care 1985; $1: 61-65$.

30. Shapiro MF, Ware JEJ, Sherbourne CD. Effects of cost sharing on seeking care for serious and minor symptoms: Results of a randomized control trial. Ann Int Med 1986; 104:246-251.

31. Haddy RI, Schmaler ME, Epting RJ. Nonemergency emergency room use in patients with and without primary care physicians. J Fam Pract $1987 ; 24: 389-392$.

32. Frey L, Schmidt J, Derksen DJ, Skipper B. A rural emergency department. West J Med 1994; 160: $38-42$.

33. Lowy A, Kohler B, Nicholl J Attendance at accident and emergency departments: unnecessary or inappropriate? J Public Health Med 1994 Jun;16(2): 134-140.

34. Afilalo M, Guttman A, Colacone A, Dankoff J, Tselios C, Beaudet M. Emergency department use and misuse. J Emerg Med 1995; 13: 259-264.

35. McLeod PJ, Meagher T, Cassidy L, Williams JI, Grover SA. Non-urgent emergency department visits by patients from a resident ambulatory care clinic. Acad Med 1995; 70:932.

36. Simon HK, Ledbetter DA, Wright J. Societal savings by (fast tracking» lower acuity patients in an urban pediatric emergency department. Am J Emerg Med 1997; 15:551-554.

37. Murphy AW, Leonard C, Plunkett PK, Bury G, Lynam F, Smith $M$ et al. Effect of the introduction of a financial incentive for fee-paying $A \& E$ attenders to consult their general practitioner before attending the $\mathrm{A} \& \mathrm{E}$ department. Fam Pract 1997; 14: 407-410

38. Restuccia JD. Appropriateness Evaluation Protocol. Manual. Barcelona: Fundación Avedis Donabedian; 1995 .

39. InterQual, Inc. The ISD-A review system with adult criteria; the ISD-A review system with pediatric criteria. Chicago, IL: InterQual, 1987.

40. SysteMetrics. Standardized Medreview Instrument: SMI: reviewer's training manual. Santa Barbara, CA: SysteMetrics-McGraw Hill; 1984. 
41. Hansagi H, Carlsson B, Olsson M, Edhag O. Trial of a method of reducing inappropriate demands on a hospital emergency department. Public Health 1987;101:99-105.

42. Hansagi H, Allebeck P, Edhag O. Health care utilization after referral from a hospital emergency department. Scand J Soc Med 1989; 17:291-299.

43. Derlet RW, Nishio DA. Refusing care to patients who present to an emergency department. Ann Emerg Med 1990; 19: 262-267.

44. Derlet RW, Nishio D, Cole LM, Silva J. Triage of patients out of the emergency department: three-year experience. Am J Emerg Med 1992; 10:195-199.

45. Prince M, Worth C. A study of (inappropriate» attendances to a paediatric Accident and Emergency Department. J Public Health Med 1992;14:177-182.

46. Eagle DJ, Rideout E, Price P, McCann C, Wonnacott $\mathrm{E}$. Misuse of the emergency department by the elderly population: Myth or reality? J Emerg Nursing 1993; 19:212-218.

47. Derlet RW, Kinser D. The emergency department and triage of nonurgent patients. Ann Emerg Med $1994 ; 23: 377-379$.

48. Derlet RW. Triage of non urgent patients out of the emergency department. Ann Emerg Med 1994; 23:204-206.

49. Derlet RW, Kinser D, Ray L. Prospective identification and triage of nonemergency patients out of an emergency department: A 5-year study. Ann Emerg Med 1995; 25:215-244.

50. Dale J, Green J, Reid F, Glucksman E. Primary care in the accident and emergency depariment: I. Prospective identification of patients. BMJ 1995; 311:423-426.

51. Murphy AW, Bury G, Plunkett PK, Gibney D, Smith M, Mullan E et al. Randomised controlled trial of general practitioner versus usual medical care in an urban accident and emergency department: process, outcome, and comparative cost. BMJ 1996; 312: 1135-1142.

52. Birnbaum A, Gallagher EJ, Utkewicz M, Gennis $\mathrm{P}$, Carter W. Failure to validate a predictive model for refusal of care to emergency-department patients. Acad Emerg Med 1994; 1:213-217.

53. Fleiss JL. The measurement of interrater agreement. En: Fleiss JL, ed. Statistical methods for rates and proportions. New York: John Wiley \& Sons; 1981.p. 212-236.
54. Hernández Aguado Y, Porta Serra M, Miralles M, García Benavides F, Bolúmar F. La cuantificación de la variabilidad en las observaciones clínicas. Med Clin (Barc) 1990; 95:424-429.

55. Strumwasser I, Paranjpe NV, Ronis DL, Share D, Shell LJ. Reliability and Validity of Utilization Review Criteria. Appropriateness Fvaluation Protocol, Standardized Medreview Instrument and Intensy-Severity-Discharge Criteria. Med Care 1990; 28: 95-111.

56. Landis JR, Koch GG. The measurement of observer agreement for categorical data. Biometrics 1977; 33: 671-679.

57. Rishpon S, Lubasch S, Epstein LM. Reliability of a method of determining the necessity for hospitalization days in Israel. Med Care 1986; 24: 279-282.

58. Santos-Eggimann B. Hospital utilization reviews under field conditions: potential and improvements. Int J Tech Assessmen Health Care 1993; 9:514-521.

59. Davido A, Nicoulet I, Levy A, Lang T. Appropriateness of admission in an emergency department: reliability of assessment and causes of failure. Qual Assur Health Care 1991; 3:227-234.

60. Peiró S, Meneu R, Roselló ML, Portella E, Carbonell R, Fernández $\mathrm{G}$ et al. Protocolo de evaluación del uso inapropiado de la hospitalización. Validación de la versión española. Med Clin 1996;107(4):124-129.

61. Booth BM, Ludke RL, Fisher EM. Reliability of a utilization review instrument in a large fiel study. Am J Med Qual 1994; 9: 68-73.

62. Inglis AL, Coast J, Gray SF, Peters TJ, Frankel SJ. Appropriateness of hospital utilitation. The validity and reliability of the Intensity Severity Discharge Review System in a United Kingdom acute hospital setting. Med Care 1995; 33: 952-957.

63. Donabedian A. Aspects of medical care administration: Specifying requeriments for health care. Cambridge, MA: Harvard University Press; 1973

64. Rosser RM, Chir B. The reliability and application of clinical judgement in evaluating the use of hospital beds. Med Care 1976; 14:39-47.

65. Coulter I, Adams A, Shekelle P. Impact of varying panel membership on ratings of appropriateness in consensus panels: a comparison of a multi- and single disciplinary panel. Health Serv Res 1995;30:577-591. 